\title{
Tangence
}

\section{La scène du livre ou le théâtre de Nathalie Sarraute}

\section{Marie Auclair}

Numéro 54, mars 1997

Poétique du Livre

URI : https://id.erudit.org/iderudit/025937ar

DOI : https://doi.org/10.7202/025937ar

Aller au sommaire du numéro

Éditeur(s)

Tangence

ISSN

0226-9554 (imprimé)

1710-0305 (numérique)

Découvrir la revue

Citer cet article

Auclair, M. (1997). La scène du livre ou le théâtre de Nathalie Sarraute.

Tangence, (54), 68-78. https://doi.org/10.7202/025937ar d'utilisation que vous pouvez consulter en ligne.

https://apropos.erudit.org/fr/usagers/politique-dutilisation/ 


\section{La scène du livre ou le théâtre de Nathalie Sarraute}

Marie Auclair

Navoir rien eu à clire et avoir voulu l'exprimer.

Jalbès, Récit

Le vide structure le jeu.

Arnatud, Freud, Wittgenstein et la musique

Une vraie pièce de thêâtre louscule le repos des sens, libère l'inconscient comprimé $[. .$.$] .$

Artaud, *Le Théâtre et lá peste.

\section{La sensation hors du lieu commun: la forme de l'absence}

Nathalie Sarraute écrivain juif: cet état de fait n'est étrangement jamais abordé par la critique. Pourtant, tout au long de son ceuvre (prose, roman, essai, théâtre), son écriture a quelque chose à voir avec la psalmodie juive et sa théâtralité intrinsèque, en ce qu'elle manifeste les lois intimes de sa production, qu'elle fait de son contenu le corps de sa lettre, qu'elle annonce l'impossibilité de son objet (référentiel) dans la texture de son énonciation ${ }^{1}$. Ce thêâtre - puisque c'est lui qui nous occupera ici ouvre donc le livre à la scène de son écriture, c'est-à-dire de son avènement; en cela, il constitue une éthique (doublée d'une poétique), le lieu de l'invention de la lettre comme oeuvre inachevée. En une parfaite circularité scripturaire déjà pressentie par la dislocation, la désintégration de l'intrigue dramatique, il met l'inviva-

1 Tout comme Artaud, Sarraute revendique l'impensable comme motif et objet de son écriture et chu passage à la théâtralisation.

2 Simone Benmussa, Nathalie Sarraute. Qui êtes-txus?, Lyon, La Manufacture, 1987, p. 15. 
ble au centre de ses revendications et "fait entendre sur scène ce que d'ordinaire on ne veut pas entendre " 2 et qu'on rejette dans l'insu. La fin de la scène, du drame et de l'écriture, y annonce toujours déjà son avènement; pour paraphraser Blanchot ${ }^{3}$, puisque le "récit" consiste en une histoire non racontable énoncée par des cris et des silences fragmentaires se subsumant en points de suspension (forme d'aphasie scripturaire). Parole - et Livre - de l'exil de mots toujours déjà exilés, de la lettre toujours déjà excentrée, cette ouvre traduit non seulement l'écart infini entre deux sujets irrémédiablement noués l'un à l'autre, mais aussi "l'espace sensible entre la lettre et la lettre" "4 dirait Jabès, où ça se tait, en souffrance et minutieusement, au coeur du dire, dans une déchirure qui se donne comme le lieu premier de l'énonciation. Là s'entend en obstinato une part d'innommé, d'originel, de réel, chutant dans un langage de conventions et d'images prises sur la ressemblance - le lieu commun, le cliché où se dissimulent les tropismes, *ces mouvements intérieurs à peine conscients et qui ne sont pas pris dans le langage " 5 . Ainsi, le réel qui "ne cesse pas de ne pas s'écrire" (Lacan) se donne à percevoir comme ponctuation du discours.

Mais le langage offre-t-il réellement un lieu commun où se transmettrait l'obscure sensation, le ressenti immédiatement intraduisible, théorisé par l'auteur dès l'écriture de Tropismes ${ }^{6}$ ? Sarraute redoute les effets de pétrification résultant de l'usage de la parole mais ne peut se soustraire à appeler le mot pour montrer l'action du tropisme? ${ }^{7}$. De l'union de la sensation et du mot, du

3 Maurice Blinchot, - L'absence de livre, Lentretien infint, Paris, Gallimard, 1969. p. 620.

4 Edmond Jalès, "La Réponse esquivée ", Limprononçable. Lécriture nomade, Paris, Collectif Change, 1975 , p. 8.

5 Nathalie Sarrate, entretien avec Arnaud Rykner, Nathalic Sarraute, Paris, Seuil, coll. - Les Contemporains a. 1991, p. 176-177.

6 Publié chez Minuit en 1957.

7 -Je prends la sensation qu'on éprouve quand quelque chose d'ignoré - on ne se l'explique pas, on ne fait pas de commentaires - est en train de se passer et aboutit à des paroles. C'est au présent. (Nathalie Sarraute, Nathalie Sarraute. Qui êtes-axuss?, p. 108) Et ces paroles sont de l'ordre de la banalité, de l'infime, de l'insignifiant (voir l'entrevue réalisée par Irène SadowskaGuillon avec Nathalie Sarraute, "À la recherche du temps présent ", Acteus, n" 34, mars 1986 , p. 15). Le cliché, le lieu commun provoxue ainsi une éruption dramatique très violente, une tragédie intérieure où l'importance des faits est inversement proportionnelle à son insigniflance sur la scène extérieure. 
réel et du symbolique, naîtra ainsi le texte, qui doit établir son équilibre entre ces deux registres. Mais cette *écriture rejette toute réponse, du fait même qu'elle est écriture, c'est-à-dire question posée à la question ${ }^{8}$, parole prise et parole donnée dans la haine de l'insupportable évidence, non pas de ce qui est dit mais d'un dire qui assassine.

Métaphoriquement, Sarraute tente par conséquent de conjoindre la représentation à un «objet*.indéterminé, à savoir la sensation, toujours déjà perdue comme présence. Le temps de l'écriture est donc celui de l'après-coup d'une reconquête perceptuelle où s'étalerait le temps trop *impulsif ", dirait Sarraute, de la sensation. La visée est d'inventer le lieu d'où l'on écrit pour authentifier la sensation comme vide, non pour la communiquer et la décrire: pour en témoigner, certes. La sensation est toujours originaire, antérieure à la lettre; cependant, puisqu'elle est leurre - et revendiquée comme tel - elle est aussi pure invention, travail de l'imaginaire, entreprise menant à la nomination. Elle n'apparaît que par le jeu d'un regard en biais porté sur les mots (des autres), sur les choses que ces mots s'essaient à véhiculer comme semblants. Elle se donne à entrevoir dans des scories, donc, des pleins en creux allégoriques 9 . La sensation, le tropisme formant une tension, un surgissement, l'écriture théâtrale tentera du coup, profitant de l'espace scénique comme lieu de l'effraction ${ }^{10}$, d'en rendre compte dans son effectuation, dans son processus d'éruption, d'intrusion.

Elle est $l \hat{a}^{11}$. Une femme (F) se tait alors qu'elle aurait pu parler; ce simple "mouvement intérieur" suffit pour faire naître chez un homme (H2) une intolérance haineuse non pas du geste mais de

8 Edmond Jabès, "La réponse esquivée *, op. cit., p. 12.

9 Sur les procédés comparatifs utilisés par Sarraute pour tenter d'inscrire la sensation, voir Rachel boué, oLieux et figures de la sensation dans l'ceuvre de Nathalie Sarratute, Littérature, n" 89, fév. 1993, p. 58-67: * L'analogie permettrait, en effet, d'exprimer cette résistance au sens et à la nomination par le détour, le transfert, le déplacement de l'innommable sur le déjà connu. P̉ar ce procédé comparatif, la fiction est suspendue à un objet impossible à nommer, mais toujours relancée vers lui. [...] Privée de la nomination et de son ancrage dans le réel, la sensation ne peut être accessible verbalement que par le clétour imagé qui en atteste à la fois la présence - l'image n'existe qu'en regard de cette sensation - et l'absence - la sensation n'appartient pas au langage $*$ (p. 60).

10 Françoise Asso, Natbalie Sarraute. Une écriture de leffraction. Paris. PUF, coll. Écrivains - 1995. $272 \mathrm{p}$.

11 Nathalie Sarratute, Fille est là, Théatre, Paris, Gallimard, [1978] 1993, p. 33-59 
l'être qui se refuse à dialoguer. La violence, ici, ne réside donc pas d'abord dans la parole offerte mais dans la parole innocemment refusée, celle qui donne forme à l'absence et à l'inassimilable.

H2: Tout à lheure, quand nous discutions... enfin, on ne peut pas appeler ça discuter, nous étions du même avis... enfin vous et moi... mais elle... elle était là quand nous parlions, elle écoutait...

H1: Qui donc? Ah, cette personne?... Votre collaboratrice?

H2: Oui... enfin... mon associée... mais peu importe qui elle est... Vous avez vu son air?

H1 : Non, à vrai clire je n’ai pas...

H2: Vous n’avez pas remarqué? Vous ne l’avez pas senti? Elle nétait pas de notre avis. Mais pas du tout...

[...]

H2: Ah, de savoir que c'est là, en elle... je ne sais pas comment vous expliquer... C'est là... Ici... (Pose deux doigts sur son front) Elle a sa petite idée... (p. 36)

Le désir de H2 n'en est que plus sollicité, relancé constamment par l'abject de ce mutisme où le sens s'effondre. Reste le manque suppurant... et la parole à en extraire... et ce désir lancinant de détruire ce manque au coeur des mots de lautre:

H2: Ce n'est pas la tête qu'il faut clétruire, c'est l'iclée... Pas le porteur... mais l'ílée qu'il porte... l'iclée seule... la traquer... l'écraser...

H3: Oui, assainir. Bien nettoyer. Faire place nette...

H2: Et alors on pourrait à cette place, dans cette même tête, installer... se répandant d'elle... se propageant...

H3: éclairant tout alutour...

$\mathrm{H} 2$ et $\mathrm{H} 3$, clans un même souffle: la vérité... (p. 52)

"Chacun est toujours en quête de ce que l'autre garde pardevers soi et la conversation sarrautienne s'apparente à une traque perpétuelle du pourquoi de l'attitude d'autrui [...]." ${ }^{12} \mathrm{La}$ haine n'en est que plus exacerbée à mesure que $F$ tente de se soustraire au discours de clichés ambiant pour nommer l'objet insu; l'inquisition repart alors en trombes, portant non plus sur

12 Arnaud Rykner, op. cit., p. 125. 
72

cet objet insu (l'idée non transmissible) mais sur l'acte de transmission même en ce qu'il démobilise l'ordre des choses et ouvre les fêlures de la loi.

Comme si c'était pour la première fois... lions-nous l'un à l'autre, pour voir encore... Renouons donc contact, engageons le dialogue, pour qu'ainsi tu me reconnaisses et que je te prenne. Essaie de me nommer, et je jouerai à t'en faire jouir... sachant d'ores et déjà que tu y échoueras. Poursuis-moi là où je ne suis pas et témoignes-en auprès de nous. Attire-moi vers toi par tes appels répétés pour que je parle et me taise pour toi, pour que le verbe en ses creux soit mon reposoir et mon calvaire. Enchaînemoi à toi comme aux nouuds de mon récit. Que ta scène soit le lieu de mon épuisement, que ta quête soit mon unité dans le réel. Ensemble, donnons lieu à la parole sur la scène, scène du Livre, mise à l'épreuve de l'origine...

\section{Là où tu es, je te hais: sacrifice et dissection de l'Autre}

C'est parce que l'autre fait défaut qu'il faut en ordonner l'autopsie... "En fait, c'est de la pratique de la lecture des autres que naîtra le livre à venir [...]." ${ }^{13}$ Ainsi, sur la scène du théâtre sarrautien, c'est parce qu'ils sont en leur essence lecteurs-interprètes anonymes ${ }^{14}$ de l'autre (et de l'Autre) que les "personnages" peuvent prendre leur place dans la communauté du Livre. "Personnage "devient en fait ici trompe-l'oil, porte-voix, sujet de l'écriture indistinct à lui-même: lieu vide et fantasmé d'une absence en acte et en corps. N'existent que des corps aliénés dans et par le regard de l'autre, miroir anihilant de l'hallucination négative où l'on ne se voit que comme absence de lettre, comme sensation furtive et non inscrite. Ainsi le corps s'en voit-il pétrifié, vidé de sa consistance par un processus dialogal correspondant à une authentique dissection langagière. Il n'y a donc ici de corps que spectral, comme sculpté au repoussé, corps-verbe constituant "l'être du mot ${ }^{15}$ plutôt que l'image du corps, se donnant à per-

13 Philippe Daros, *Les Livres des autres ou soi-même comme un autre •, Litterature, $\mathrm{n}^{\circ} 92$, déc. 1993 , p. 70.

14 Il faut savoir que dans ce théâtre, l'usage du nom propre est marginalisé au profit de l'usage du pronom ou de la simple lettre.

15 Lucette Finas, * Nathalie Sarraute ou les métamorphoses du verbe *, Tel quel, $\mathrm{n}^{\circ} 20,1965$, p. 68. 
cevoir dans le même geste où il se dérobe: sacrifice du corps... et du nom. Les "personnages" ne sont de fait qu'un lieu à travers lequel passe la parole: ils ne forment que la somme de leurs énonciations aliénantes, et non de leurs énoncés - ce qui les ancrerait socialement ailleurs que dans cette neutralité qui leur octroie leur statut.

Parce que l'écriture sarrautienne tient du cérémonial d'expiation ${ }^{16}$ et expose une structure obsessionnelle soumettant la moindre tentation d'agir (de parler ou de se taire) à un châtiment envisagé et même espéré, se lisent les traces du refoulement d'une pulsion de violence et de mort repérables dans l'énonciation de la haine. Lire l'autre, faire comme si on entendait en son *langage essentiel" le lieu même de sa profération, permet de détourner cette pulsion destructrice et d'ainsi ne pas se soumettre au refoulement par l' incorporation du signifiant lui-même " ${ }^{17}$ comme objet. Changement de but, sublimation: lecture se renversant en écriture par simple riposte.

La violence, dans l'écriture de Nathalie Sarraute, est conjointe à l'attente et au danger, à la menace de se voir offrir en réponse un relancement vers l'ailleurs (et un retour à la case départ), vers l'interminable épreuve de l'altérité (et de l'aliénation). Profération et profanation en sont deux modes d'existence exemplaires. Sur cette scène, ce qu'il s'agit de faire, c'est d'interroger la marge: la question signale donc obstinément l'errance du sujet, le déchiffrement d'une absence, puisqu'elle est parole en attente de ce Livreénigme dont l'écriture forme à la fois l'évitement. La question, la nomination, le jugement, constituent l'engramme de l'événement, et en cela deviennent eux-mêmes événement. Ce qui se passe ici, ce n'est rien d'autre que la conversation, qui ne fait que signaler des corps déstabilisés, sidérés, dessaisis, incrédules d'être épelés comme lettre muette, offerts ainsi aux aléas de l'interprétation qui exhorte à témoigner de l'impossible rencontre entre deux sujets dans le discours - comme de l'impossible rencontre entre la sensation et la lettre. Tout se joue dans une dynamique d'incorporation de l'autre: l'attaque porte alors sur la fissure dans la langue de l'autre, sur un *objet langagier" qui fait tache et iclentifie,

16 Voir Gérard Haddad, Manger le Livre. Rites alimentaires et fonction paternelle, laris, Grasset, coll. * Figures *, 1984, p. $20 \mathrm{sq}$.

17 Jacques Lucan, Le séminaire, livre VII: Léthiqué de la psychanalyse, Paris, Seuil, coll. "Le chimp freudien., 1986, p. 340. 
statufie. Ne reste ensuite qu'à tenter de s'extirper de cet étranglement sournois et assassin. Parce qu'alors le "personnage" est mis en boîte, il s'en troúve à la fois étiqueté et clésidentifié. Il s'agit bel et bien d'une haine de l'être, de l'Autre dans sa cléchéance face au sens: en somme, c'est parce qu'il y a trop d'autre qu'il faut l'exterminer. La scène du Livre se construit donc à partir des rapports de négativisation s'ancrant sur la tyrannie du mot. Par exemple, la nomination, résultat du jugement du désir de l'un désir insu donc violé - par les mots de l'autre, signe l'exclusion, la réduction, et non la marque de l'appartenance. Partant, une fonction épuratoire est à reconnaître ici dans l'acte de nommer, qui signale de même une sorte de délire de persécution à l'ouvre. La structure de base de ce drame est donc celle de l'exclusion de celui qui sert de bouc-émissaire, c'est-à-dire de celui qui refuse le drame de la parole comme drame, tel une bête prise au piège et qui refuserait d'en sortir.

\section{Le Livre du (presque) rien : passages, passions et variations}

Le Livre comme présence-absence infinie dépeint une compulsion de répétition morbide, puisque la question n'appelle pas la réponse mais bien ce moment où elle tombera dans le vide (l'Autre) du continent inconnu qu'est le langage de la dépossession. Ce qui motive cette écriture, ce n'est pas l'objet du manque mais bien le manque d'objet, qui se révèle purement fascinant; ce manque est objet, du fantasme et non du désir puisqu'il le cause, objet insensé et en fuite opérant dans le réel - puiqu'au fond, ce qui est désiré c'est la dit-mension du réel, ce qui se trouve hors-jeu, ce qui borde le symbolique. Témoignage "d'un secret sans contenu, sans contenu séparable de son expérience performative, de son tracement performatif " 18 , cette écriture se supporte d'un (presque) rien qui ne relèverait pas d'une intériorité privée, intériorité qu'il faudrait à tout prix dévoiler ou confesser. En fait, Sarraute par son éthique de l'écriture laisse entendre qu'on a droit au secret et que celui-ci est partageable, puisqu'il fait problème et force au dire; toutefois, ce secret qui n'est pas un phénomène demeure en fin de parcours inexpugnable et irréductible à sa nomination, car il se pose là contre, hors temps et

18 Jacques Derrida, Passions, laris, Galilée, 1993, p. 56. 
hors lieu comme motif de la scène d'écriture, du Livre et de l'Autre.

Liber: accouchement, délivrance, libération. Si l'on en croit Sarraute, rien de plus incertain: "À peine cette chose informe, toute tremblante et flageolante, cherche-t-elle à se montrer au jour qu'aussitôt ce langage si puissant et si bien armé, qui se tient prêt à intervenir pour rétablir l'ordre - son ordre - saute sur elle et l'écrase. "19 La scène du Livre n'est donc pas scène de libération mais scène de lutte extrême. Nathalie Sarraute ne met donc rien d'autre en scène: elle met le rien en livre, sur le mode de l'aria da capo, du motif et de la variation, puisqu'elle ramène inlassablement la particule inaperçue et mortifère pour la réviser. Chez elle, l'espace scénique n'est rien d'autre que l'espace d'engendrement du Livre, celui de la Loi de l'Autre où la parole est donnée ${ }^{20}$ et mise en activité. Partant, le drame est soumis aux lois de la fragmentation et de la pulsation, c'est-à-dire du temps de l'intervalle qui rythme une rencontre qui n'advient jamais réellement. En effet, la sensation et le mot sont irrémédiablement disjoints - ce qui évacue toute possibilité d'instauration d'un système sémiotique où se construirait le sens. La rencontre de l'autre n'advient jamais, dans l'acte d'écriture, que comme la manifestation pérenne d'une faillitte de recouvrement temporel et de réparation de l'altération de la lettre: ce qui était à inscrire n'est pas encore advenu réellement mais le peut-il seulement? - sur cette scène là, celle de l'écriture, où ce qui reste encore inimaginé fait basculer le sujet dans un nonsavoir qui le contrarie et le fait se sentir coupable de ne pas être (ou demeurer) conforme à une certaine image de soi. Or, cette image idéale est telle parce que détruite par le mot de l'autre, motscansion qui épelle et juge son vis-à-vis et le suspend au-dessus du vide. Ce que Sarraute écrit et dépeint, c'est justement ce suspens. du bic et nunc comme épreuve du dire, naissance à soi et à l'autre qui l'appelle dans l'espoir d'une rencontre, invariablement déceptive, où le sujet est convié à dire ce qu'il ne sait pas.

Partant, deux entités scéniques - nous ne pouvons plus parler ici de "personnages" - , deux mises en formes de la lettre se

19 Nathalie Sarraute, "Ce que je cherche à faire ", Nouteau Roman: hier, aujourd hui. Tome II: Pratiques, Paris, U.G.E., coll. *10/18*, 1972, p. 37.

20 C'est en ce sens que l'écriture sarrautienne est une recherche éthique, en ce qu'elle ouvre la parole comme lieu cl'une texture à venir. lieu cle l'Autre où le sujet est joué et rejoué. 
76

regardent, s'observent, se scrutent et s'interprètent. Aussitôt conçoivent-ils qu'ils sont vus en train de voir (regard autoréflexif) qu'ils sont pris de panique: "[...] jeu cle miroir où je me perds mon image que je projette en lui ou celle qu'il plaque aussitôt, férocement, sur moi, comme fait l'autre $[\ldots]_{n} 21$. Jeu de miroir qu'ils ont chacun son tour instauré. Un regard myope dépossède, aliène: scène de ravissement où l'autre aspire l'un hors de luimême et le rend indistinct, hors de toute reconnaissance, mais fixé dans un mot sàns issue où se perd le sens comme devenir. À cet instant précis de sa "nomination" - le plus souvent adjectivale et péjorative - l'un se sait vu pas l'autre en ce lieu précis d'où il regarde et constate la fissure. Demeure un inévitable clivage entre la représentation de soi et l'intimité de soi en tout ce qu'elle draîne d'inavouable. *Et c'est de la sujétion à ce regard des autres dont souffrent les personnages de Nathalie Sarraute; ce regard dont ils ne peuvent se passer, ce regard qui leur donne forme et à laquelle ils s'identifient, et qui n'est pourtant que source de tromperie [...]. ${ }^{22}$ Tromperie, mensonge: imposture où un regard qui n'a rien d'autre à exposer que son manque tente de se poser comme Vérité et d'imposer à l'autre une posture qui le clouerait sur place. La brèche est alors ouverte - c'est le temps du tropisme, temps originaire et mythique - qu'il s'agira non de colmater mais d'articuler, dans un travail de ressassement de la souffrance en tant qu'elle s'écrie, rejettant ainsi l'objet de discussion loin des zones de déploientent de la mémoire, là où aurait pu advenir quelque chose comme du nom propre.

"Qui est à l'outure dans le vis-à-vis? Qui parle? Qui regarde? Question inexpugnable, où le désir se porte mais ne se fixe. Cercle impensable de ce retour à la ressemblance, à la nécessité blanche de communiquer. "23 "Parole irrécupérable, parce qu'elle porte au-delà de tout discours, bors les mots ${ }^{24}$, scission où ne se reconnaissent plus ni le sujet ni l'objet, signalant la faillite du discours au profit des mécanismes servant à défendre un vide de sens. Cette parole de l'autre "dit l'impossible, ne nommant rien que la distance à ce rien (nous soulignons). [...] Parole dévidée,

21 Nathalie Sarraute, Martereau, Paris, Gallimard, coll. "Folio *. 1953, p. 241.

22 Rachel Boué, op. cit., p. 64.

23 Jacques Sojcher, * L'atute parole*, Cabiers internationaux de symbolistne, $\mathrm{n}^{\prime 2 \mathrm{~s}} 19-20,1970$, p. 57.

24 Ibid., p. 58 
épuisée - forage intérieur exténuant - vouée à la clépense des formes $[\ldots]^{25}$. Ne reste qu'à écouter l'autre et à tenter cle forger sur des bases passives un lieu commun. Cette écriture se fait donc création de formes par une exigence de figurabiblité. En cela, elle constitue un moyen de lutter, et avec quel acharnement, contre l'angoisse de la présence de l'informe, inscrivant un corps de lettre là où est entrevue une dissolution identitaire. Sacrifiant un corps graphique, elle clonne lieu à la fiction et répond à l'insoutenable. L'acte scénique, clans ce théâtre, est donc un acte oral de reconduction de la question: d'interprétation, de partage du dire. Par une écriture implosive, la matière discursive - l'objet chu, c'est-à-dire la sensation - disparaît dans le vicle qui en est le coeur; les mots y sont partant pris à témoin du passage de la jouissance et de la souffrance de l'Autre et par l'Autre. Courant éperdument après son hien-dire et sa Vérité, le "personnage" porte-voix s'élance après l'assentiment de l'Autre; ainsi se voit-il indubitablement confronté à la nécessité vitale et incontournable de traduire (presque en justice) la cassure interne de la loi qui fait que le dire est donné en pâture dans le dialogue, réelle traversée du déjà-dit au profit du dire du sujet, dire qui vient à s'écrire sur un dire antérieur, commun et fantasmé, qui jamais n'exista. Puisque ça n'était pas, ça doit se répéter, dira-t-on avec Lacan. La répétition résulte donc d'une rencontre du manque, puisqu'il s'agit de rejouer en mots une scène originaire qui n'a pas eu lieu, de rendre palpables un lieu et un temps insus, qui consistent en la rencontre du sujet et de son désir ${ }^{26}$. Bien évidemment, cette rencontre est toujours manquée. le temps de la scène n'est que celui de la parole, donc celui du désir en acte comme un battement de cil filmé et vu au ralenti, avec des arrêts sur image permettant de grossir les modulations scéniques de la pause/pose, moment où l'un décortique l'autre pour mieux faire voir au-delà de ce que l'oeil a coutume de distinguer. Ce qui est montré, ce sont les rouages du désir et du passage à l'acte qu'est la parole sur cette scène transactionnelle faite de méprises, de délibérations, de jugements, de reprises, etc. C'est pourquoi le dialogue se fait parfois polyphonie ou même monologues parallèles mais toujours interdépendants, temps visible de la plainte et clu récitatif, expérience concrète du ralentissement, permettant d'entendre

25 Ibid., p. 59.

26 . Le désir fonde l'histoire comme temps du sujet qui advient. " Denis Vasse, La chair ennisagée. La génération spmbxlique, 1'aris, Seuil, 1988. p. 7. 
78

les pulsations d'une vocalisation/vocifération amplifiée et rythmée par la souffrance résultant de la rupture persistante de la lettre. Ce temps réclusif est aussi, paradoxalement, temps infini de la répétition du même, c'est-à-dire du ratage: or, ce temps-là n'offre aucune catharsis. Faire parler l'Autre comme "passage cle Lettre, lieu de transit pour la Chose qui s'inscrit "27, dialoguer avec le Livre sur la scène de l'écriture: voilà qui pourrait résumer le théâtre de Nathalie Sarraute. Le Livre est donc à concevoir comme lieu du poétique, en ce que "le poétique, c'est ce qui parle dans le langage, de la part de l'Autre, dans le partage du Dire" 28.

27 Daniel Sibony, Jonissances du dire. Nondeanx essais sur whe transmission dincomicient, Paris, Grasset et Falsquelte, coll. "Figures", 1985, p. 12.

28 Ihid.. p. 60. 